BMJ Open

Diabetes

Research

\& Care

\title{
Culture-centered approaches: the relevance of assessing emotional health for Latinos with type 2 diabetes
}

\author{
Jeannie Belinda Concha, ${ }^{1,3}$ Briana Mezuk, ${ }^{1}$ Bonnie Duran ${ }^{2,3}$
}

To cite: Concha JB, Mezuk B, Duran B. Culture-centered approaches: the relevance of assessing emotional health for Latinos with type 2 diabetes. BMJ Open Diabetes Research and Care 2015;3: e000064. doi:10.1136/ bmjdrc-2014-000064

- Additional material is available. To view please visit the journal (http://dx.doi.org/ 10.1136/bmjdrc-2014000064).

Received 2 October 2014 Revised 4 August 2015 Accepted 9 August 2015

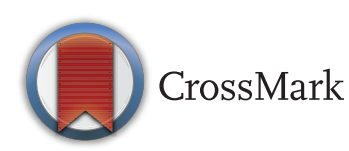

${ }^{1}$ Division of Epidemiology, Department of Family Medicine and Population Health, Virginia Commonwealth University, Richmond, Virginia, USA ${ }^{2}$ University of Washington, School of Social Work, Seattle, Washington, USA ${ }^{3}$ Indigenous Wellness Research Institute, National Center of Excellence, Seattle Washington, USA

Correspondence to Dr Jeannie Belinda Concha; jbconcha@vcu.edu

\section{ABSTRACT}

Objective: Within Latino culture, there is a belief that strong emotions can cause diabetes. Because of this belief and evidence regarding the bi-directional relationship between depression and diabetes, the objectives of this study were to determine if medical doctors are asking Latinos with diabetes about emotional problems and to assess attitudes toward professional help for emotional problems.

Research design and methods: Data come from the nationally representative National Latino and Asian American Study and the National Comorbidity Survey Replication study. Only Latino subsamples were included $(n=3076)$. A smaller subsample with complete data $(n=2568)$ was used for the inquiry outcome variable. Weighted $\chi^{2}$ analysis and logistic regression were conducted to determine the likelihood of being asked about emotional problems and attitudes toward professional help.

Results: Latinos with mood disorders or anxiety (MD/ $\mathrm{AX} ; \mathrm{OR} 2.84,95 \% \mathrm{Cl} 2.02$ to 4.00 ), diabetes only (OR $1.69,95 \% \mathrm{Cl} 1.06$ to 2.69$)$, and co-occurring diabetes and MD/AX (OR 6.67, 95\% Cl 2.33 to 19.04) were more likely to be asked about emotional problems, relative to Latinos without diabetes or MD/AX. A minority of respondents with diabetes $(32 \%)$ were asked about emotional problems. Respondents with diabetes only were more likely to feel comfortable talking to a professional for personal problems compared with those without diabetes or MD/AX (OR $1.44,95 \% \mathrm{Cl} 0.99$ to 2.09 ). Although the relationship between having diabetes and feeling comfortable taking to a professional is not statistically significant, z-test statistics indicate that having diabetes influences attitudes about discussing emotional problems.

Conclusions: Among Latinos, having diabetes is associated with greater likelihood of being asked about emotional problems and feeling comfortable talking to a professional about personal problems. Consistent with the cultural relevance of emotions as a cause of diabetes, asking about emotional problems may be a useful approach for engaging Latinos into a discussion about their diabetes self-care activities.

\section{INTRODUCTION}

Type 2 diabetes is a complex and multifaceted disease that is often associated with substantial emotional distress. Approximately

\section{Main research points}

- Type 2 diabetes disproportionately impacts the growing USA Latino population, both in terms of incidence, disease management (eg, glycemic control and self-management), complications, and diabetes-related emotional distress.

- Within Latino culture, there is a belief that strong emotions can cause diabetes, thus addressing the emotional status of Latinos with diabetes may be culturally relevant.

- Findings indicate that Latinos with diabetes are more likely to be asked about emotional problems than those without diabetes and suggestive evidence that diabetes is associated with more positive attitudes about talking to a professional about emotional problems.

\section{Prospective research questions}

- For healthcare providers: Does entering into a discussion about emotional stress improve the understanding of diabetes self-care recommendations among Latinos?

- Does improving the emotional adjustment to diabetes improve glycemic outcomes among Latinos?

$40 \%$ of individuals with diabetes experience clinically significant diabetes-related emotional distress. ${ }^{1}$ The co-occurrence of type 2 diabetes and emotional problems is particularly important for the Latino population. Latino groups in the USA are disproportionality affected by type 2 diabetes. Compared with the national estimate $(9.3 \%)$, the prevalence of diabetes in Latinos ranges between $12 \%$ and $19 \%$, with substantial variation across Latino subgroups. ${ }^{2} 3$ The lifetime prevalence of anxiety is strongly associated with diabetes among Latinos, ${ }^{34}$ and diabetesrelated distress is higher in this group compared with Caucasians and AfricanAmericans. ${ }^{5}$ As Latinos continue to be one of the fastest growing ethnic minority groups in the USA, it is expected that type 2 
diabetes will continue to be a serious emotional and health burden for this group. ${ }^{6}$ Thus, addressing and engaging Latinos with culturally appropriate healthcare approaches is of great importance. ${ }^{7-9}$

One approach for addressing diabetes care among Latinos is to use a culture-centered approach for enhancing the way diabetes information is communicated and received. ${ }^{10}$ Culture-centered is a health communication approach that is based 'on the commitment to building theories and applications from within the culture' and is guided by using a cultural lens for understanding a patient's own theory about their disease and how they make meaning of it in the context of their lives. ${ }^{10}$ Culture-centered approaches may be particularly useful for Latinos with diabetes because, within Latino culture, there is a historical culture-centered belief that strong or negative emotions can cause diabetes and suboptimal glycemic levels. ${ }^{11-19}$ This belief is known as susto or 'fright sickness' and many Latinos continue to hold this belief. ${ }^{11-19}$ Poss and Jezeweski ${ }^{19}$ use the following quote in their research to illustrate how emotions influence diabetes: "Well, perhaps a person starts to feel very depressed and kind of weak and sick, and then, little by little, diabetes starts to develop in the body. That is what I think. And then the person starts to feel uneasy and nervous, and he eats more and more of the wrong type of foods. The only symptom I had was that I felt kind of sick and had no strength" (p.370).

Given that, within Latino culture, emotions are believed to be an important aspect of diabetes, asking Latinos about their diabetes causation beliefs, emotional health, and how it affects their diabetes management may be a useful communication approach for medical clinicians. Culture-centered approaches can provide clinicians with a unique opportunity and a first step toward engaging Latinos in a meaningful and practical discussion about diabetes risks, health promotion behaviors, and diabetes self-care activities. ${ }^{20-23}$ Likewise, medical clinicians can benefit from understanding the role of emotional problems in diabetes management, given the established relationship between symptoms of emotional health problems (eg, anxiety and depression), coping, and self-regulatory health behaviors that impact glycemic control (eg, high glycemic/carbohydrate food choices, tobacco use, physical inactivity). ${ }^{24-30}$

In addition to the benefit of improving glycemic control, inquiring about emotional problems can lessen the stigma and alleviate reservations about discussing problems like depression or anxiety. Because depression or anxiety among Latinos is often masked with somatic symptoms rather than symptoms used within standard mental health diagnostic criteria, Latinos tend to rely on primary care and general medicine for mental healthcare and treatment. ${ }^{22} 30$ The presence of a physical illness, like diabetes, may lessen the stigma of having emotional problems and allow Latinos to be more open with medical doctors about their emotional status because they can attribute it to their physical condition.
The purpose of this paper is to illuminate recommendations by the American Diabetes Association to routinely screen a patient for psychosocial problems 'at diagnosis, during regularly scheduled management visits, during hospitalizations, with the discovery of complications, or when problems with glucose control, quality of life, or self-management are identified'. ${ }^{31}$ Thus, the primary objective of this study is to determine the extent to which medical doctors are asking Latinos with diabetes about their emotional problems. The secondary objective of this study is to compare attitudes toward professional help for emotional problems between Latinos without diabetes or mood/anxiety disorders $(\mathrm{MD} / \mathrm{AX})$ and those with $\mathrm{MD} / \mathrm{AX}$ only, diabetes only, and co-occurring diabetes and MD/AX.

We hypothesize that medical doctors are less likely to ask Latinos with diabetes about emotional problems compared with those without diabetes or MD/AX. We hypothesize a crowd-out effect, which proposes that the competing demands of physical conditions monopolizes medical doctors' time and attention, consequently deprioritizing care for patients emotional health. ${ }^{32}$ We also propose that a medical doctor's unfamiliarity with Latino culture-centered diabetes causation theories influences the likelihood of asking Latinos with diabetes about emotional problems. While crowd-out effect is not derived from a culture-centered framework, this hypothesis can be used as a proxy to assess for features of culturecentered approaches. For example, in this paper, we investigate whether medical doctors are asking Latinos with diabetes about emotional problems as an underlying feature of culture-centered approaches. Second, we hypothesize that Latinos with diabetes will be more likely to have positive attitudes toward professional help for their emotional problems versus those without diabetes or $\mathrm{MD} / \mathrm{AX}$, because they can minimize stigma associated with emotional problems and attribute it to having diabetes.

\section{METHODS}

The data for this study come from the National Latino and Asian American Study (NLASS) and the National Comorbidity Survey Replication (NCS-R). Both NLASS and NCS-R are part of the Collaborative Psychiatric Epidemiologic Studies (CPES), three US national mental health surveys. ${ }^{33} 34$ Adults 18 years and older were recruited using multistage stratified sampling of nationally representative households and high-density supplemental sampling for the inclusion of racial/ ethnic minority groups. Interviews for these studies were conducted between 2001 and 2003 and in the respondents' preferred language (eg, English or Spanish). Interviews were conducted in person or by telephone by trained interviewers from the Institute for Social Research at the University of Michigan. As part of the NLAAS and NCS-R interviews, respondents completed the World Mental Health Composite International 
Diagnostic Interview (WMH-CIDI), a fully structured diagnostic interview designed to identify psychiatric disorders as defined by the Diagnostic and Statistical Manual of Mental Disorders-IV. ${ }^{35}$ Additional details about the study design and survey methodology for NLAAS are described elsewhere. ${ }^{33}{ }^{34}$ CPES is approved by the Institutional Review Board at the University of Michigan and all participants provided informed consent. This analysis is limited to Latino respondents who reported having diabetes or not $(\mathrm{N}=3076)$. The sample primarily includes individuals from Mexican $(\mathrm{N}=1210)$, Puerto Rican $(\mathrm{N}=494)$, Cuban $(\mathrm{N}=576)$, and other Latino ( $\mathrm{N}=796)$ backgrounds. Because Latino subgroups were not substantially different across diabetes status and the primary outcome variable, we aggregated Latinos into one group for analysis. A smaller subsample with complete data was used in the $\chi^{2}(\mathrm{n}=2568)$ and logistic regression analysis $(n=2565)$ of the inquiry outcome variable. The ages of the analytic sample ranged between 18 and 98 years (mean=39.8).

\section{Measures}

\section{Diabetes status}

Diabetes status was assessed by self-report of physician diagnosis, combined with age of diagnosis. Among the respondents with diabetes, $7 \%(\mathrm{~N}=14)$ reported being told they have diabetes prior to age 20, thus presumably have type 1 diabetes. Because the purpose of this study is to determine the frequency and attitudes toward professional help for emotional problem among adult Latinos with diabetes, this subset of respondents with type 1 diabetes was retained in the analysis.

\section{Mental health status}

History of $\mathrm{MD} / \mathrm{AX}$ was determined using the WHM-CIDI. ${ }^{36}$ Diagnoses made with the WMH-CIDI have moderate concordance with clinical psychiatric interviews. ${ }^{37}$ For this analysis, we created a dichotomous variable $(\mathrm{MD} / \mathrm{AX})$ to indicate the presence of any of the following four DSM-IV MD/AX in the past 12 months: dysthymia, major depressive episode (MDE), generalized anxiety disorder (GAD), and post-traumatic stress disorder (PTSD). Mental health status was also captured based on self-reported perception of their status (eg, excellent, very good, good, fair/poor).

\section{History of mental health services utilization}

Lifetime history of mental health services use for 'emotional problems, substance use, or nerves' was indexed by two dichotomous variables: one that indicated history of psychological counseling and another that indicated history of using psychiatric medications.

\section{Sociodemographic characteristics}

Age (in years), sex, marital status (categorized as married/cohabitating), household income (categorized as \$16 999 or less, $\$ 17000-\$ 44999, \$ 45000-\$ 79999$, and $\$ 80000$ and over) educational attainment (categorized as 11 or less, 12, 13 years or more) were assessed by self-report.

\section{Outcomes}

\section{Emotional status inquiry by medical doctor}

Respondents were asked, "In the past 12 months, did a medical doctor ask you about your emotions, nerves, or mental health?" and responses were assessed using a binary (yes/no) variable.

\section{Attitudes toward professional help for emotional problems}

Attitudes regarding the use of professional help for emotional problems were assessed using the following three questions: (1) If you had a serious emotional problem, would you definitely go for professional help, probably go, probably not go, or definitely not go for professional help? (2) How comfortable would you feel talking about personal problems with a professional and (3) How embarrassed would you be if your friends knew you were getting professional help for an emotional problem? Each item was assessed using a Likert scale, and the responses were then recoded into dummy variables (eg, $1=$ definitely go/probably go, $0=$ probably not go/definitely not go, and $1=$ very comfortable/somewhat comfortable, $0=$ not very comfortable/not at all comfortable).

\section{Statistical analysis}

Respondents were categorized into four mutually exclusive groups: (1) no diabetes and no MD/AX in the past 12 months (ie, dysthymia, GAD, MDE, PTSD; reference group), (2) no diabetes but at least one $\mathrm{MD} / \mathrm{AX}$ in the past 12 months, (3) current diabetes but no MD/AX in the past 12 months, and (4) comorbid diabetes with MD/AX.

Initially, the characteristics of the sample across the four-level diabetes/MD variable were compared using Scott-Rao $\chi^{2}$ tests. The sample for income $(\mathrm{n}=3075)$ and self-rated mental health $(\mathrm{n}=2645)$ was limited to respondents who endorsed these items. Next, a series of logistic regression models were fit to assess the association between diabetes and the two outcomes: clinician inquiry of emotional problems and attitudes toward mental health services use (ie, three items addressing willingness, comfort, and embarrassment). We examined whether concurrent MD/AX impacted this relationship using the four-level variable described above. Regressions were adjusted for gender, age, county of origin, income, education, and marital status. For each logistic regression analysis, the sample was restricted to respondents with complete data for the main exposure variables, outcome variables, and relevant covariates (ie, for the analysis of emotional health inquiry, all models have a sample size of 2565; for the analysis of willingness to seek professional help, all models have a sample size of 3073; for the analysis of comfort, all models have a sample size of 3066; for the analysis of embarrassment, all models have a sample size of 3066). 
Finally, we conducted a post hoc analysis to test whether the parameter estimates across the health status groups (MD/AX only vs diabetes only, MD/AX only vs diabetes+MD/AX, and diabetes only vs diabetes $+\mathrm{MD}$ / $\mathrm{AX})$ were significantly different using z-tests. ${ }^{38}$ Significant z-tests indicate heterogeneity in the $\beta$-coefficients in these comparisons, even if the estimates themselves are not significantly different from the null. Significant z-tests for the MD/AX only versus diabetes comparisons, in conjunction with non-significant z-tests for the diabetes only versus diabetes+MD/AX comparisons, are consistent with our hypotheses that diabetes influences attitudes about discussing emotional problems for Latinos, perhaps because of cultural health beliefs like susto and the connections between emotions and physical health.

All analyses were weighted using Stata IC V.11 using survey procedures and the cpeswtsh weighting variable to account for the study design. Respondents with missing data on the exposure, outcome, and covariate variables were excluded from the adjusted analysis. All $\mathrm{p}$ values refer to two-tailed tests.

\section{RESULTS}

The lifetime prevalence of diabetes among Latinos was $8 \%$, and the 12-month prevalence of a $\mathrm{MD} / \mathrm{AX}$ was $11 \%$. Table 1 shows the distribution of demographic and health characteristics by the four groups of diabetes and MD/AX status. Consistent with previous research, older age was positively associated with diabetes and inversely associated with presence of a MD/AX in the past 12 months $\left(\chi^{2}=28.13, \mathrm{p}<0.001\right)$. Latinos with diabetes were less likely than those without diabetes to report 'excellent' or 'very good' mental health $\left(\chi^{2}=10.9\right.$, $\mathrm{p}<0.001)$. Among the Latinos respondents in this sample, 427 were identified with at least one MD/AX. MDE was the most common 12-month mood disorder among both individuals with diabetes (47\%) and without diabetes $(67 \%)$.

Table 2 illustrates the relationship between the diabetes and MD/AX groupings, medical doctor's inquiry of emotional health, and attitudes toward professional help for emotional problems. Among the 2568 cases with complete data used for this analysis, the probability of medical doctors asking about emotional problems was highest for Latinos with co-occurring diabetes and $\mathrm{MD}$ / AX (59\%) and Latinos with MD/AX only $(36 \%$ : $\left.\chi^{2}=21.43, \mathrm{p}<0.00\right)$. Among Latinos with diabetes, $32 \%$ were asked about emotional problems. The probability of willingness to see a professional for help with emotional problems was highest for Latinos with diabetes only $(90 \%)$ and those with neither condition $(86 \%$ : $\left.\chi^{2}=4.64, \mathrm{p}<0.00\right)$. With regard to feeling comfortable talking about personal problems with a health professional, Latinos with diabetes only $(83 \%)$ and co-occurring diabetes+MD/AX $(79 \%)$ were more likely to feel comfortable talking about their personal problems compared with those with neither condition (72\%) and those with MD/AX only (68\%: $\chi^{2}=5.76$, $\mathrm{p}<0.00)$. Although the comparison was only marginally significant, Latinos with diabetes only $(16 \%)$ were less likely to be embarrassed about getting professional help for emotional problems compared with those with MD/ AX only (26\%), those with co-occurring diabetes and $\mathrm{MD} / \mathrm{AX}(24 \%)$, and those with neither condition (22\%: $\left.\chi^{2}=2.63, \mathrm{p}=.07\right)$.

Online supplementary figure 1 displays history of mental health services utilization by $\mathrm{MD} / \mathrm{AX}$ and diabetes status. Among 644 respondents who were asked these utilization questions, 153 reported ever using either psychological counseling or medications for emotional problems, drug use, alcohol use, nerves, or emotions. Psychological counseling in combination with medications was the most common form of treatment every used for those with MD/AX (79\% for MD/AX only, and 59\% with MD/AX+diabetes). Medication use was most common in those with diabetes only $(52 \%)$. Latinos with neither condition reported lowest utilization of medications (37\%), psychological counseling $(32 \%)$, and their combination $(31 \%)$.

Table 3 shows the unadjusted and adjusted association between the combinations of diabetes/mental health status (ie, diabetes only, diabetes+MD/AX, and MD/AX only) on the two outcomes: inquiry of emotional problems by a medical doctor and attitudes toward professional help for emotional problems. Among the 2568 respondents with complete data, those with diabetes only (OR $1.69,95 \%$ CI 1.06 to 2.69 ) were more likely to be asked about emotional problems compared with those without diabetes or MD/AX. Likewise, those with MD/ AX (OR 2.84, 95\% CI 2.02 to 4.00) and co-occurring diabetes and MD/AX (OR 6.67, 95\% CI 2.33 to 19.04) were three and seven, respectively, times as likely to be asked about emotional problems compared with those with neither condition. The estimate for co-occurring diabetes $+\mathrm{MD} / \mathrm{AX}$ is less precise because it reflects the small number of cases in this group. With regard to attitudes toward professional help, Latinos with MD/AX only were less willing to see a professional for serious emotional problems compared with those without diabetes and without $\mathrm{MD} / \mathrm{AX}$ (OR $0.50,95 \%$ CI 0.35 to 0.70 ). Although it did not reach statistical significance, respondents with diabetes were more likely to feel comfortable talking to a professional for emotional problems compared with those without diabetes or MD/AX (OR 1.44, $95 \%$ CI 0.99 to $2.09, \mathrm{p}=0.054$ ).

In a post hoc analysis, we further examined the contrasting relationships between diabetes and $\mathrm{MD} / \mathrm{AX}$ on the four outcomes by comparing parameter estimates across three groups (MD/AX only vs diabetes only, MD/AX only vs diabetes+MD/AX, and diabetes vs diabetes+MD/AX) using z-tests. There was significant heterogeneity in the relationship between $\mathrm{MD} / \mathrm{AX}$ only versus diabetes only among the emotional inquiry outcome $(\mathrm{Z}=1.84, \mathrm{p}=0.033)$, willingness to seek professional help outcome $(\mathrm{Z}=-1.96$, 
Table 1 Sample characteristics by diabetes and MD/AX status $(\mathrm{N}=3076)$

\begin{tabular}{|c|c|c|c|c|c|c|c|c|c|}
\hline & \multicolumn{2}{|c|}{$\begin{array}{l}\text { No diabetes or } \\
\text { MD/AX } \\
\mathrm{N}=2439(83 \%)\end{array}$} & \multicolumn{2}{|c|}{$\begin{array}{l}\text { MD/AX only } \\
N=371(9 \%)\end{array}$} & \multicolumn{2}{|c|}{$\begin{array}{l}\text { Diabetes only } \\
\mathrm{N}=210(7 \%)\end{array}$} & \multicolumn{3}{|c|}{$\begin{array}{l}\text { Diabetes+MD/AX } \\
\mathrm{N}=56(1 \%)\end{array}$} \\
\hline & $\mathbf{n}$ & $(\%)$ & $\mathbf{n}$ & $(\%)$ & $\bar{n}$ & $(\%)$ & $\mathbf{n}$ & $(\%)$ & p Value \\
\hline \multicolumn{10}{|l|}{ Country of origin } \\
\hline Cuban & 462 & (4) & 59 & (5) & 42 & (5) & 13 & (6) & 0.07 \\
\hline Puerto Rican & 368 & (7) & 65 & (11) & 46 & (11) & 15 & $(17)$ & \\
\hline Mexican & 965 & (66) & 149 & (61) & 78 & (63) & 18 & (53) & \\
\hline Other Latino & 644 & (23) & 98 & (23) & 44 & (21) & 10 & (24) & \\
\hline \multicolumn{10}{|l|}{ Age (years) } \\
\hline $18-34$ & 1127 & (51) & 181 & (54) & 24 & (11) & 9 & $(16)$ & 0.00 \\
\hline $35-49$ & 781 & (31) & 108 & (28) & 49 & (23) & 21 & (35) & \\
\hline $50-64$ & 370 & (12) & 58 & (12) & 76 & (36) & 20 & (38) & \\
\hline 65 or more & 161 & (6) & 24 & (6) & 61 & (30) & 6 & (11) & \\
\hline Female & 1323 & $(45)$ & 253 & (56) & 121 & (56) & 42 & (62) & 0.00 \\
\hline Married/cohabitating & 1541 & (63) & 199 & (52) & 122 & (66) & 26 & $(47)$ & 0.00 \\
\hline \multicolumn{10}{|l|}{ Income $(\$)^{\star}$} \\
\hline $0-16999$ & 657 & (29) & 123 & (33) & 82 & (37) & 31 & $(45)$ & 0.259 \\
\hline $17000-44999$ & 819 & (34) & 117 & (29) & 63 & (28) & 12 & (19) & \\
\hline $45000-79999$ & 517 & (21) & 71 & (22) & 39 & (22) & 10 & $(24)$ & \\
\hline $80000+$ & 446 & (16) & 59 & (16) & 26 & (13) & 3 & (12) & \\
\hline \multicolumn{10}{|l|}{ Education (years) } \\
\hline 11 or less & 858 & $(42)$ & 134 & (38) & 97 & (51) & 35 & $(57)$ & 0.278 \\
\hline 12 & 651 & (28) & 92 & (27) & 44 & (23) & 8 & (16) & \\
\hline 13 or more & 930 & (30) & 145 & (35) & 69 & (26) & 13 & $(27)$ & \\
\hline \multicolumn{10}{|c|}{ Self-rated mental health* } \\
\hline Excellent & 729 & (32) & 51 & (19) & 46 & (24) & 1 & $(4)$ & 0.00 \\
\hline Very good & 628 & (29) & 67 & (25) & 37 & (21) & 5 & $(10)$ & \\
\hline Good & 591 & (29) & 95 & (33) & 69 & (38) & 8 & $(20)$ & \\
\hline Fair/poor & 175 & (10) & 76 & (23) & 36 & (17) & 31 & $(66)$ & \\
\hline \multicolumn{10}{|l|}{ 12-month MD/AX } \\
\hline Dysthymia & & & 3 & (1) & & & 2 & $(4)$ & 0.00 \\
\hline Generalised anxiety & order & & 33 & (8) & & & 8 & $(15)$ & \\
\hline Major depressive ep & & & 240 & (67) & & & 30 & $(47)$ & \\
\hline Post-traumatic stres & order & & 95 & (24) & & & 16 & (34) & \\
\hline \multicolumn{10}{|c|}{ Mean years with diabetes (SE) } \\
\hline & NA & & NA & & 9.5 & 43) & & 83) & \\
\hline
\end{tabular}

$\mathrm{p}=0.025)$, comfort talking to professional outcome $(\mathrm{Z}=-2.21, \mathrm{p}=0.014)$, and embarrassed for getting professional help outcome $(\mathrm{Z}=1.92, \mathrm{p}=0.027$; table 3$)$. These findings indicate that relative to respondents with $\mathrm{MD} / \mathrm{AX}$ only, Latinos with diabetes had more positive attitudes about mental health services, even though they were less likely to be asked about their emotional health by their physician. There was no heterogeneity in the parameter estimates comparing MD/AX only versus diabetes $+\mathrm{MD} /$ $\mathrm{AX}$; and the z-tests for the diabetes only versus diabetes $+\mathrm{MD} / \mathrm{AX}$ were marginally significant for inquiry about emotional status and attitudes about seeking help, but not attitudes about comfort or embarrassment. Taken together, these findings are consistent with the hypothesis that the presence of diabetes, but not $\mathrm{MD} / \mathrm{AX}$, is related to more positive attitudes about mental health services among Latinos.

\section{DISCUSSION}

The findings of this study indicate that Latinos with diabetes, with $\mathrm{MD} / \mathrm{AX}$, or these conditions in combination are more likely to be asked about emotional problems compared with those without these conditions. Latinos with diabetes only were more likely to feel comfortable talking to a medical doctor about personal problems relative to those without diabetes or MD/AX. Taken together, our results suggest that diabetes is associated with more positive attitudes about mental health services among Latinos. However, our findings also show that Latinos, regardless of their health status, are only rarely asked about their emotions by medical providers.

Our study shows no evidence of the hypothesized crowd-out effect, whereby the presence of comorbid diabetes reduces the likelihood that healthcare providers will ask about emotional health needs. As a whole, our 
Table 2 Diabetes and mood/anxiety status by probability of emotional health inquiry and attitudes toward professional help for emotional problems

\begin{tabular}{|c|c|c|c|c|c|c|}
\hline \multicolumn{7}{|c|}{ Emotional health inquiry by medical doctor } \\
\hline \multicolumn{7}{|c|}{ In the past 12 months, did a medical doctor ask about your emotions, nerves, or mental health? } \\
\hline & \multirow[b]{2}{*}{$\mathrm{N}=2568$} & \multicolumn{2}{|l|}{ No } & \multicolumn{2}{|l|}{ Yes } & \multirow{2}{*}{$\begin{array}{l}\chi^{2} \\
p \text { Value }\end{array}$} \\
\hline & & $\overline{\mathbf{N}}$ & $(\%)$ & $\overline{\mathbf{n}}$ & $(\%)$ & \\
\hline No diabetes or MD/AX & 1994 & 1601 & (84) & 393 & $(16)$ & 0.00 \\
\hline MD/AX only & 327 & 94 & (64) & 133 & (36) & \\
\hline Diabetes only & 197 & 139 & (73) & 58 & (27) & \\
\hline Diabetes+MD/AX & 50 & 22 & (41) & 28 & (59) & \\
\hline No diabetes & 2321 & 1795 & (81) & 526 & (19) & 0.00 \\
\hline Diabetes & 247 & 161 & (68) & 86 & (32) & \\
\hline
\end{tabular}

\section{Attitudes about professional help for emotional problems}

\begin{tabular}{|c|c|c|c|c|c|c|}
\hline & \multirow[b]{2}{*}{$N=3076$} & \multicolumn{2}{|c|}{$\begin{array}{l}\text { Probably not/ } \\
\text { definitely not }\end{array}$} & \multicolumn{2}{|c|}{ Definitely/probably } & \multirow[b]{2}{*}{0.00} \\
\hline & & $\bar{n}$ & $(\%)$ & $\bar{n}$ & $(\%)$ & \\
\hline No diabetes or MD/AX & 2439 & 299 & (14) & 2140 & (86) & \\
\hline MD/AX only & 371 & 75 & (23) & 296 & (77) & \\
\hline Diabetes only & 210 & 20 & (10) & 190 & (90) & \\
\hline Diabetes+MD/AX & 56 & 8 & (21) & 48 & (79) & \\
\hline
\end{tabular}

\section{How comfortable would you feel talking about personal problems with a professional?}

\begin{tabular}{|c|c|c|c|c|c|c|}
\hline & \multirow[b]{2}{*}{$N=3066$} & \multicolumn{2}{|c|}{ Not very/not at all } & \multicolumn{2}{|c|}{ Very/somewhat } & \multirow[b]{2}{*}{0.00} \\
\hline & & $\overline{\mathbf{n}}$ & $(\%)$ & $\overline{\mathbf{n}}$ & $(\%)$ & \\
\hline No diabetes or MD/AX & 2432 & 610 & (28) & 1822 & (72) & \\
\hline MD/AX only & 371 & 108 & (32) & 263 & (68) & \\
\hline Diabetes only & 207 & 38 & (17) & 169 & (83) & \\
\hline Diabetes+MD/AX & 56 & 16 & (21) & 40 & (79) & \\
\hline
\end{tabular}

How embarrassed would you be if your friends knew you were getting professional help for an emotional problem?

\begin{tabular}{|c|c|c|c|c|c|c|}
\hline & \multirow[b]{2}{*}{$N=3069$} & \multicolumn{2}{|c|}{ Not very/not at all } & \multicolumn{2}{|c|}{ Very/somewhat } & \multirow[b]{2}{*}{0.07} \\
\hline & & $\bar{n}$ & $\overline{(\%)}$ & $\bar{n}$ & $\overline{(\%)}$ & \\
\hline No diabetes or MD/AX & 2433 & 1959 & (78) & 474 & (22) & \\
\hline MD/AX only & 371 & 286 & (74) & 85 & (26) & \\
\hline Diabetes only & 209 & 179 & (84) & 30 & (16) & \\
\hline Diabetes+MD/AX & 56 & 42 & (76) & 14 & (24) & \\
\hline
\end{tabular}

Values are unweighted frequency counts and weighted percentages. $\mathrm{MD} / \mathrm{AX}, \mathrm{mood} / \mathrm{anxiety}$ disorder.

findings provide partial support to our second hypothesis regarding the relationship between diabetes and positive attitudes toward professional help for emotional problems. We hypothesize this because the presence of a physical illness, like diabetes, may lessen the stigma of emotional problems among Latinos, particularly in light of cultural health beliefs like susto which focuses on the impact of stress and emotions on physical health. ${ }^{11-19}$ The results of our post hoc analyses were consistent with this interpretation, with the pattern of results indicating that diabetes specifically is related to positive attitudes toward mental health services.

Although previous literature indicates that the stigma concerning psychiatric disorders can keep individuals from seeking mental health service care, particularly for
Latinos, ${ }^{22} 39$ our findings indicate that Latinos feel comfortable speaking with a professional about their emotional status, which is one indication of receptivity to mental health services. Decades of research has demonstrated the importance of beliefs regarding strong or negative emotions and diabetes within Latino culture. ${ }^{11-19}$ Previous studies have also emphasized the role of stress and emotions in diabetes self-care, ${ }^{40}$ but often these experiences are treated as secondary to issues of diabetes education and health behavior change. The presence of diabetes can provide an opportunity to discuss the emotional status of Latino patients and how it influences diabetes self-care activities.

With respect to diabetes healthcare, we believe that inquiring about the emotional status of Latinos with 
Table 3 Association between diabetes and mental health status on likelihood of emotional health inquiry and attitudes toward professional help for emotional problems

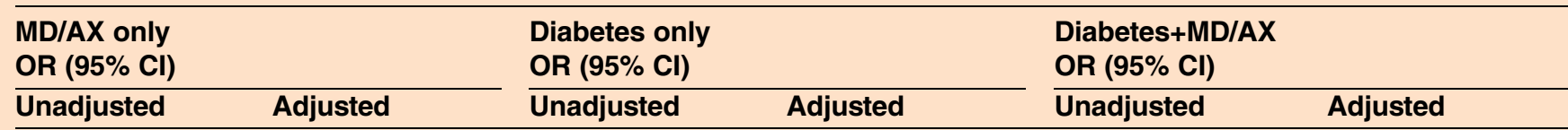

Emotional health inquiry by medical doctor

In past 12 months, did a medical doctor ask about your emotions, nerves, or mental health? (N=2565)

2.94 (2.13 to 4.08$) \quad 2.84$ (2.02 to 4.00$) \quad 1.91$ (1.27 to 2.89$) \quad 1.69$ (1.06 to 2.69$)^{\star} \quad 7.49(2.83$ to 19.84$) \quad 6.67$ (2.33 to 19.04$)$

Attitudes about professional help for emotional problems

If you had a serious emotional problem, would you go for professional help? $\quad(\mathrm{N}=3073)$

0.53 (0.37 to 0.75$) \quad 0.50$ (0.35 to 0.70$) \quad 1.45(0.76$ to 2.76$) \quad 1.39(0.73 \text { to } 2.65)^{*} \quad 0.62$ (0.21 to 1.78$) \quad 0.56(0.20$ to 1.58$)$

How comfortable would you feel talking about personal problems with a professional? $\quad(\mathrm{N}=3066)$

0.84 (0.67 to 1.05$) \quad 0.81$ (0.65 to 1.02$) \quad 1.97$ (1.40 to 2.75$) \quad 1.44$ (0.99 to 2.09$)^{\star} \quad 1.49$ (0.61 to 3.62$) \quad 1.31$ (0.54 to 3.18$)$

How embarrassed would you be if your friends knew you were getting professional help for an emotional problem? (N=3066)

1.23 (0.95 to 1.59$) \quad 1.25$ (0.94 to 1.66$) \quad 0.66(0.43$ to 1.03$) \quad 0.78(0.51 \text { to } 1.19)^{*} \quad 1.11$ (0.52 to 2.33$) \quad 1.33(0.57$ to 3.11$)$

Reference, no diabetes or MD/AX.

Adjusted for gender, age, country of origin, income, education, and marital status.

${ }^{\star} z$-Test, $p<0.05 \mathrm{MD} / \mathrm{AX}$ only versus diabetes only.

$\mathrm{MD} / \mathrm{AX}$, mood/anxiety disorder.

diabetes may benefit medical clinicians in their communication approach toward establishing diabetes management goals with this group. Prior work has indicated that the psychosocial relationship between a medical clinician and a Latino patient is viewed as an essential aspect in gaining trust and improving adherence to recommended self-care activities or treatments. ${ }^{25}$ Thus, incorporating discussions about emotional well-being and identifying psychosocial stressors as management barriers can improve patient self-care activities by enhancing the patient-provider relationship and communication by personalizing the relationship (otherwise known as 'personalismo'). ${ }^{39}$ Asking Latinos with diabetes about emotional problems can help engage the patient and personalize diabetes treatments in a way that addresses psychosocial issues. Consequently, incorporating discussions around the culture-centered diabetes causation theories, like strong emotions causing diabetes, may help establish goals that are more salient for the patient.

We recognize the caveats of emotional health assessment for Latinos in the USA: health providers have limited time during patient visits, mental health services for this group are neither readily available or accessible, and the quality of these services may be inadequate or culturally insensitive. ${ }^{30}$ However, based on our study, Latinos with diabetes do report that medical providers are asking about emotional problems, while still infrequently, more often than they are for Latinos who do not have either diabetes or MD/AX conditions.

Findings should be interpreted in light of study limitations. Diabetes status and mental health services use was assessed by self-report. Biological data were not available to validate diabetes status. Since diabetes is often underdiagnosed, particularly for Latinos, this indicates that our findings are likely underestimates. In addition, we restricted our analysis to respondents with complete data for all the exposure and outcome variables, including covariates, thus limiting the use of the entire Latino sample. This study also has a number of strengths. NLAAS is the only nationally representative, populationbased sample of Latino mental health, and this design enhances the generalizability of the findings and mitigates selection bias. MD/AX were assessed using a wellvalidated diagnostic instrument. Finally, we were able to examine multiple aspects of mental health services use.

In conclusion, this study indicates that having diabetes increases Latinos' likelihood of being asked about emotional status, and provides suggestive evidence that the presence of diabetes is associated with more positive attitudes about mental health services. Given these results and the Latino health belief that strong emotions can cause diabetes, addressing emotional health may be a novel culturecentered clinical communication approach for discussions that are 'responsive to the patient's preferences, needs and values'. 31 Thus, asking Latinos with diabetes about their emotional status may provide clinicians with greater opportunity to engage with these patients and establish diabetes treatment goals that are culturally appropriate.

Author's note For the purpose of this paper we use the term Latino to be sensitive and inclusive of all Latin American ethnic groups and nationalities represented in the National Latino and Asian American Study. We recognise that the term Hispanic is often interchangeable with Latino; however, because it is a term that was instituted in the USA and is associated with European Spanish we decided to exclusively use Latino throughout this paper (Alcof L. Latino vs. Hispanic: the politics of ethnic names. Philos Soc Criticism 2005;9:10-21).

Contributors JBC conceptualized researched data and wrote the manuscript. BM contributed to the analysis plan and reviewed/edited the manuscript. $\mathrm{BD}$ reviewed and contributed to the introduction and discussion. 
Funding The research was supported in part by the Center for Disease Control and Prevention (CDC) Training Program Grant (1 T01 CD000189-01). AHRQT32 Training Grant HS013853-06. BM is supported by a NIH (K01-MH093642 and R21-DK8356430).

Competing interests None declared.

Provenance and peer review Not commissioned; externally peer reviewed.

Data sharing statement The data used for this study are available through the Inter-university Consortium for Political and Social Research (ICPSR) at the University of Michigan.

Open Access This is an Open Access article distributed in accordance with the Creative Commons Attribution Non Commercial (CC BY-NC 4.0) license, which permits others to distribute, remix, adapt, build upon this work noncommercially, and license their derivative works on different terms, provided the original work is properly cited and the use is non-commercial. See: http:// creativecommons.org/licenses/by-nc/4.0/

\section{REFERENCES}

1. Nicolucci A, Kovacs Burns $\mathrm{K}$, Holt Rl, et al. Diabetes Attitudes, Wishes and Needs second study (DAWN2 ${ }^{\mathrm{TM}}$ ): cross-national benchmarking of diabetes-related psychosocial outcomes for people with diabetes. Diabet Med 2013;30:767-77.

2. Daviglus M, Talavera G, Aviles-Santa L, et al. Prevalence of major cardiovascular risk factors and cardiovascular disease among Hispanic/Latino individuals of diverse backgrounds in the United States. JAMA 2012;308:1775-84.

3. Mier N, Bocanegra-Alonso A, Zhan D, et al. Clinical depressive symptoms and diabetes in a binational border population. $J A m$ Board Fam Med 2008;21:223-33.

4. Ortega A, Feldman J, Canino G, et al. Co-occurrence of mental and physical illness in US Latinos. Soc Psychiatr Epidemiol 2006:41:927-34.

5. Spencer MS, Kiefer EC, Sinco BR, et al. Diabetes-specific emotiona distress among African Americans and Hispanics with type 2 diabetes. J Health Care Poor Underserved 2006;17:88-105.

6. Boyle J, Thompson T, Gregg E, et al. Projection of the year 2050 burden of diabetes in the US adult population: dynamic modeling of incidence, mortality, and prediabetes prevalence. Popul Health Metr 2010;8:29.

7. Cusi K, Ocampo G. Unmet needs in Hispanic/Latino patients with type 2 diabetes mellitus. Am J Med 2011;124(10 Suppl):S2-9.

8. Kahn LS, Fox CH, McIntyre RS, et al. Assessing the prevalence of depression among individuals with diabetes in Medicaid managed-care program. Int J Psychiatr Med 2008;38:13-29.

9. Centers for Disease Control and Prevention. 2011 National Diabetes Fact Sheet. http://www.cdc.gov/diabetes/pubs/estimates11.htm\#4

10. Dutta MJ. Communicating about culture and health: theorizing culture-centered and cultural sensitivity approaches. Commun Theory 2006;17:304-28.

11. Cherrington A, Ayala GX, Sleath B, et al. Examining knowledge, attitudes, and beliefs about depression among Latino adults with type 2 diabetes. Diabetes Educ 2006;32:603-13.

12. Arcury TA, Skelly AH, Gesler WM, et al. Diabetes meanings among those without diabetes: explanatory models of immigrant Latinos in rural North Carolina. Soc Sci Med 2004;59:2183-93.

13. Daniulaityte R. Making sense of diabetes: cultural models, gender and individual adjustment to type 2 diabetes in a Mexican community. Soc Sci Med 2004;59:1899-912.

14. Satterfield DW, Lofton T, May JE, et al. Learning from listening common concerns and perceptions about diabetes prevention among diverse American populations. J Public Health Manag Pract 2003:9:S56-63.

15. Mercado-Martinez FJ, Ramos-Herrera IM. Diabetes: the layperson's theories of causality. Qual Health Res 2002;12:792-806.
16. Alcozer F. Secondary analysis of perceptions and meanings of type 2 diabetes among Mexican American women. Diabetes Educ 2002;26:785-95.

17. Weller SC, Baer RD, Pachter LM, et al. Latino beliefs about diabetes. Diabetes Care 1999;22:722-8.

18. Rubel AJ, O'Nell CW, Collando-Ardo R. Susto, a folk Illness. Berkeley, Los Angeles, CA: University of California Press, 1984

19. Poss J, Jezewski MA. The role and meaning of susto in Mexican American's explanatory model of type 2 diabetes. Med Anthropol $Q$ 2002;16:360-77.

20. Caballero AE. Building cultural bridges: understanding ethnicity to improve acceptance of insulin therapy in patients with type 2 diabetes. Ethn Dis 2006;16:559-68.

21. Cabassa LJ, Hansen MC, Palinkas LA, et al. Azucar y nervios: explanatory models and treatment experiences of Hispanics with diabetes and depression. Soc Sci Med 2008;66:2413-24.

22. Concha J, Kravitz H, Chin M, et al. Review of type 2 diabetes management interventions for addressing emotional well-being in Latinos. Diabetes Educ 2009;35:941-58.

23. Peek M, Cargill A, Huang E. Diabetes health disparities: a systematic review of health care interventions. Med Care Res Rev 2007;64(5 Suppl):101S-56S.

24. Gross T, Olfson M, Gameroff MH, et al. Depression and glycemic control in Hispanic primary care patients with diabetes. J Gen Intern Med 2005;20:460-6.

25. Li C, Barker L, Ford ES, et al. Diabetes and anxiety In US adults: findings from the 2006 Behavioral Risk Factor Surveillance System. Diabet Med 2008;25:878-81.

26. Li C, Ford ES, Strine TW, et al. Prevalence of depression among US adults with diabetes. Diabetes Care 2007;31:105-7.

27. Ali S, Stone MA, Peters JL, et al. The prevalence of comorbid depression in adults with diabetes: a meta-analysis. Diabetes Care 2006;24:1069-78.

28. Gonzalez JS, Safren SA, Cagliero E, et al. Depression, self-care, and medication adherence in type 2 diabetes. Diabetes Care 2008;30:2222-7.

29. Mezuk B, Abdou C, Hudson D, et al. "White Box" epidemiology and the social neuroscience of health behaviors: the environmental affordances model. Soc Ment Health 2013;3:79-95.

30. Cabassa LJ, Zayas LH, Hansen MC. Latino adults' access to mental health care: a review of epidemiological studies. Adm Policy Ment Health 2006;33:316-30.

31. American Diabetes Association. Standards of medical care in diabetes 2015. Diabetes Care 2015;38(S1):S20-30.

32. Klinkman MS. Competing demands in psychosocial care: a model for the identification and treatment of depressive disorders in primary care. Gen Hosp Psychiatry 1997;19:98-111.

33. Alegria $M$, Takeuchi $D$, Canino $G$, et al. Considering context, place and culture: the National Latino and Asian American Study. Int $J$ Methods Psychiatr Res 2004;13:208-20.

34. Heeringa S, Wagner J, Torres M, et al. Sample designs and sampling methods for the Collaborative Psychiatric Epidemiology Studies (CPES). Int J Methods Psychiatr Res 2004;13:221-40.

35. Kessler R, Merikangas K. The National Comorbidity Survey Replication (NCS-R). Int J Methods Psychiatr Res 2004;13:60-8.

36. Kessler R, Ustun T. The World Mental Health (WMH) survey initiative version of the World Health Organization Composite International Diagnostic Interview (CIDI). Int J Methods Psychiatr Res 2004;13:93-121.

37. Eaton WW, Hall AL, Macdonald R, et al. Case identification in psychiatric epidemiology: a review. Int Rev Psychiatry 2007; 19:497-507.

38. Paternoster R, Brame R, Mazerolle $\mathrm{P}$, et al. Using the correct statistical test for the equality of regression coefficients. Criminology 1998;36:859-66.

39. Lipton RB, Losey LM, Giachello AL, et al. Attitudes and issues in treating Latino patients with type 2 diabetes: view of healthcare providers. Diabetes Educ 1998;24:67-71.

40. Norris SL, Nichols PJ, Caspersen CJ, et al. Increasing diabetes self-management education in community settings. A systematic review. Am J Prev Med 2002;22(4 Suppl):39-66. 